scientific figure and a professional writer.

Because the history of popular science has been studied only recently and has concentrated largely on Victorian Britain, there is little to compare it with in terms of other periods or countries. But the large number of editions of Jane Marcet's various Conversation books from the early nineteenth century - and not discussed by Lightman, being pre-Victorian - indicates that there could have been a steady growth in science books before the Victorian boom.

Lightman has only one chapter on how scientific information was displayed, and uses images simply to enliven his text. By contrast, every image Ralph O'Connor uses advances his argument on the popularization of just one science, palaeontology. What Lightman gains in breadth, O'Connor makes up in depth.

O'Connor integrates the many genres that made fossils popular in the nineteenth century, using images from newspapers, books, magazines and pamphlets - including a striking one from 1828, where books were arranged to look like geological strata (pictured) - as well as John Martin's paintings, lecture illustrations, displays, dioramas and panoramas (for advertisements and handbills). O'Connor shows that promoting knowledge about geology was then similar to the marketing of other types of literature and art - science was an integral part of culture.

Books such as these, and Peter Bowler's eagerly anticipated history of popular science literature during the first half of the twentieth century, have much to offer today's debate about science education and engagement. Many of the 'public understanding of science' initiatives launched in the 1980s came to grief when the 'real' rather than the 'evaluative' world intervened. Looking back at the Royal Society's 1985 report on the subject, one wonders whether some historical perspective might have helped. History, full of contingency as it is, will not, of course, repeat itself. But the present boom in scholarship on the history of popular science should ensure that we come to appreciate our predecessors' efforts.

Frank A. J. L. James is president of the British Society for the History of Science and professor of the History of Science at the Royal Institution, 21 Albemarle Street, London W1S 4BS, UK. $\mathrm{He}$ is author of Christmas at the Royal Institution.

\section{Pulling power}

\section{The Universal Force: Gravity, Creator of Worlds \\ by Louis A. Girifalco \\ Oxford University Press: 2007. 288 pp.} E19.99

\section{Sean Carroll}

Gravity, the weakest known force, is the most obvious in our everyday life. The urge to understand it has challenged generations of great physicists, from Galileo and Newton to Einstein and Hawking. Yet gravity remains aloof and mysterious. The attempt to reconcile it with quantum mechanics is one of the most ambitious and urgent programmes of modern physics. Small wonder popular books on the topic - such as Louis Girifalco's addition - have an enduring appeal.

Our understanding of gravitation is encapsulated in Einstein's general theory of relativity. This supposes that space and time together form a dynamical four-dimensional manifold whose curvature influences the motion of matter. Girifalco tackles this well-trodden ground in the time-honoured way: by focusing on the historical development of the concepts and the colourful scientists involved.

Scientists today typically share a distorted and oversimplified view of the development of their subject, passed down through shared anecdotes of a series of brilliant insights and heroic discoveries. The reality is understandably messier and Girifalco weaves an interesting narrative from the complex history of this field. He opens with an extended discussion of Newton before introducing the ancient Greeks and the Copernican revolution.

Girifalco's historical focus allows him to include material rarely covered in other books on gravity. For example, he digresses to contrast the personalities and skills of Michael Faraday and James Clerk Maxwell and the development of modern electromagnetism - the first true field theory that builds a crucial bridge between

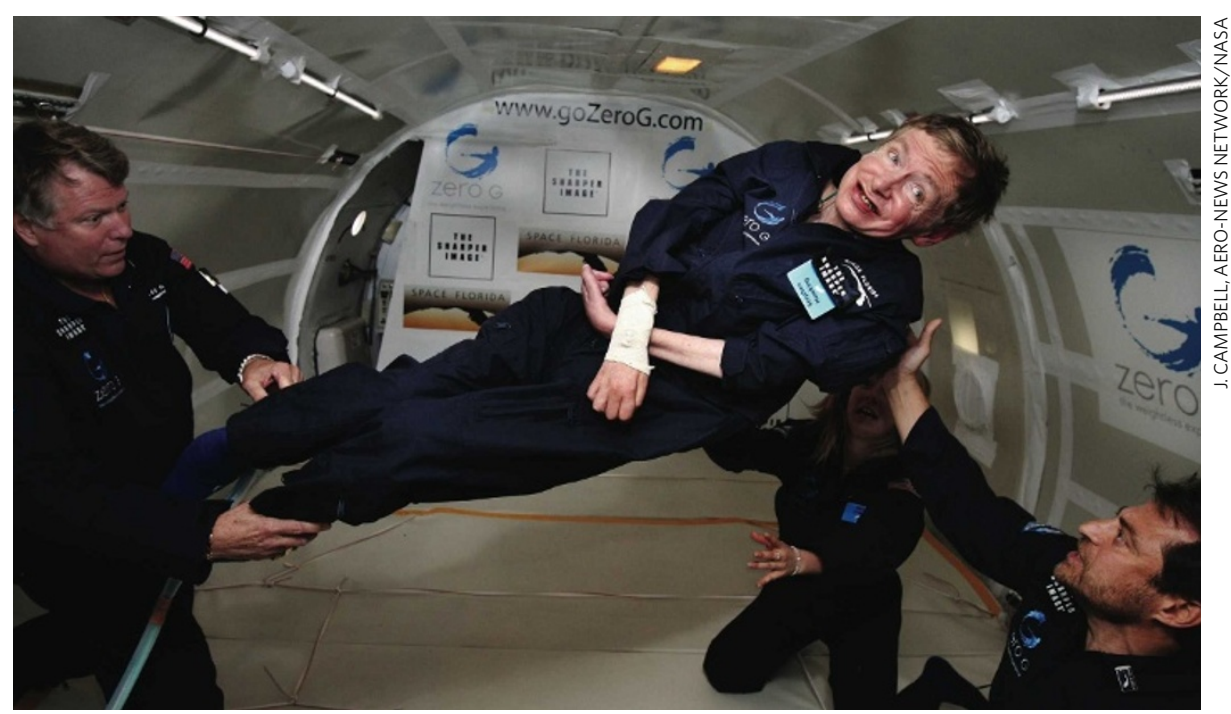

Even to Stephen Hawking - here enjoying zero gravity in a jet - the force remains mysterious.

newtonian gravity and relativity.

The Universal Force fills a niche. Many people who might be interested in physics can be turned off by its abstraction, and physicists have an unfortunate predilection for explaining their subject to non-experts by simply watering down the explanations they would give to their students. For anyone interested in the more human side of science, this work is a valuable contribution.

The emphasis on storytelling over concepts, however, creates pedagogical challenges. For instance, the book is free of pictures and diagrams: a puzzling omission. A picture of the patterns made by iron filings in the presence of a magnet would have helped explain Faraday's lines of magnetic force. Referring to the bending of light by curved space-time, the author writes: "There is a picturesque two-dimensional model that can help our understanding." Shame we don't get to see it.

Modern gravitational physics is not the author's specialty - he is a solid-state theorist - and it shows in the sections on general relativity and curved space-time. Girifalco is correct to emphasize that general relativity is essentially a very simple theory, its intimidating reputation notwithstanding. But it is harder than he makes it out to be in saying, "the laws of physics are the same for everybody, everywhere". Properly interpreted, this motto could equally well apply to newtonian gravity.

Similarly, many of the most exciting aspects of general relativity get short shrift. Black holes and the Big Bang each get a brief chapter, and there is almost nothing about the thrilling prospects for gravitational waves. Hawking's epochal (if theoretical) discovery that black holes emit radiation is a mere footnote, and no mention of string theory sullies the pages. Yes, these subjects have been thoroughly picked over in other books, but a reader expecting an introduction to some of Einstein's more recent progeny will feel cheated.

Caveats aside, Girifalco is a fluid writer, and his stories are compelling. This book about the force of gravity has its feet firmly on the ground.

Sean Carroll is a senior research associate in physics at the California Institute of Technology, 1200 E. California Blvd, Pasadena, California, USA. He is author of Spacetime and Geometry. 